\section{Patogénesis y manejo actual de la enfermedad de Kawasaki}

Pathogenesis and current management of Kawasaki disease

\section{RESUMEN}

La enfermedad de Kawasaki es una morbilidad febril aguda caracterizada por presentar vasculitis multisistémica aún desconocida, con mayor predominio en niños menores de 5 años, causa aneurismas de las arterias coronarias con complicaciones y secuelas como cardiopatía isquémica e infarto agudo de miocardio si no recibe tratamiento oportuno. Entre los factores patogénicos destacan los genéticos y ambientales. $\mathrm{El}$ tratamiento con inmunoglobulina intravenosa reduce el riesgo de desarrollar aneurismas de las arterias coronarias, además del uso de aspirina y glucocorticoides. Aquí, describimos la patogenia de la enfermedad de Kawasaki y discutimos el progreso obtenido frente a la terapéutica.

PALABRAS CLAVE: kawasaki, manejo patogenesis, tratamiento 
addition to the use of aspirin and glucocorticoids. Here, we describe the pathogenesis of Kawasaki disease and discuss the progress made versus therapeutics.

KEYWORDS: Kawasaki disease, pathogenesis, therapeutics, treatment.

\section{INTRODUCCIÓN}

La enfermedad de Kawasaki (EK) es una vasculitis aguda autolimitada que afecta a vasos de pequeño y mediano calibre y es la causa más común de enfermedad cardiaca adquirida en niños (1), clínicamente se caracteriza por la aparición fiebre, conjuntivitis bilateral no purulenta, eritema labial y oral, cambios en las extremidades, exantema y adenopatías laterocervicales (2), (3), sin embargo, el diagnóstico de EK en pacientes menore de un año de edad es un reto diagnóstico con presentaciones clínicas incompletas y mayor riesgo de desarrollar complicaciones cardiacas graves (4).

Las principales complicaciones son cardiacas y entre ellas los aneurismas coronarios, estos pueden llevar a muerte súbita, infarto agudo de miocardio (IAM) y arterioesclerosis prematura (5); quiere decir que, sin tratamiento, hasta $25 \%$ desarrolla ectasias o aneurismas coronarios, con riesgo de infarto agudo al miocardio y muerte súbita (6).

Ante la sospecha de EK debe iniciarse siempre tratamiento, aunque se tenga infección confirmada microbiológicamente (7). El tratamiento depende de la gravedad y extensión de las lesiones coronarias. (8), no obstante, basada en la mejor evidencia para los proveedores de atención médica que diagnostican y manejan la enfermedad de Kawasaki, pero la toma de decisiones clínicas debe individualizarse según las circunstancias específicas del paciente, ya que puede conducir a complicaciones graves como aneurismas de las arterias coronarias y oclusiones tromboembólicas (9).

En este contexto, se pretende revisar y actualizar el manejo actual de la enfermedad de Kawasaki a fin estratificar pautas terapéuticas según a los registros publicados en los últimos cinco años.

\section{MATERIALES Y MÉTODOS}

Este estudio de revisión y actualización sobre la enfermedad de Kawasaki sobre su terapéutica, mediante una búsqueda exhaustiva de datos secundarios y terciarios en las bases de datos. Se emplearon buscadores como "kawasaki" y "manejo" o "patogenesis" y "tratamiento". Se analizaron todos los estudios publicados entre enero a marzo 2016 a 2020, publicados en español, inglés. Entre los buscadores literarios empleados están: Google académico, PubMed, Scopus, Scielo, Up to date, Latindex, y Web of Science. 


\section{RESULTADOS}

Los aneurismas de las arterias coronarias se desarrollan en algunos niños con enfermedad de Kawasaki que no reciben tratamiento y provocan cardiopatía isquémica e infarto de miocardio (10) entonces, EK es ahora la causa más común de cardiopatía adquirida en los niños (11) que, requiere seguimiento riguroso con pruebas de esfuerzo cardíaco e imágenes no invasivas para detectar estenosis progresiva, trombosis y oclusión luminal que pueden conducir a miocardio (12). Las complicaciones cardíacas representan la principal causa de morbimortalidad tanto en la fase aguda como subaguda de la enfermedad (13).

El diagnóstico de EK es cada vez más frecuente y con un reconocimiento precoz y un tratamiento oportuno, el desarrollo de aneurismas coronarios se ve disminuido. (14). Un diagnóstico y tratamiento precoz dentro de los 10 días del inicio de la fiebre permite una reducción de las alteraciones coronarias en estos pacientes (15).

Es importante descartar otras patologías que relacionen síntomas similares para esto se realizan controles de laboratorio para descartar otras enfermedades, en la biometría se evidencia la leucocitosis, trombocitopenia, anemias hemolíticas, inflamación son signos de la enfermedad de Kawasaki, así el aumento de SGOT, SGPT, fosfatasa alcalina y bilirrubina; piuria estéril con ligera proteinuria y microhematuria; pleocitosis moderada en LCR; Cultivos negativos. Las pruebas para detectar el péptido natriurético tipo B (BNP) liberado cuando el corazón está bajo estrés pueden ser útiles para diagnosticar la enfermedad de Kawasaki.

Por otro lado, la ecocardiografía es la modalidad de imagen de elección para la detección de anomalías de las arterias coronarias y la evaluación de la función miocárdica. También es útil para la caracterización y estratificación del riesgo de pacientes con EK, en fase aguda puede mostrar alteraciones (disminución de la fracción de eyección y aneurismas a partir de la 1a semana y sobre todo a las 3-4 semanas) (16).

\begin{tabular}{|c|c|c|c|}
\hline Aspirina & GGIV & Glucocorticoides & otros \\
\hline Dosis inicial 80 & admin & Los corticoides están & Si $r$ \\
\hline $100 \mathrm{mg} / \mathrm{kg} /$ día en & gar & cor & las \\
\hline 3-4 dosis, durante & intravenosa (IGIV) a & tratamiento & coronaria, \\
\hline 2 semanas & razón de $2 \mathrm{~g} / \mathrm{kg}$, en & bargo, & Aspirina se añadirá \\
\hline hasta 48 horas & dosis & pacientes & dipiridamol a 3-5 \\
\hline después de que el & lentamente ( $d$ & responden bien a la IGIV & $\mathrm{mg} / \mathrm{kg} /$ día \\
\hline paciente quede & de la infusión: 10 & se ha pr & sodilata \\
\hline afebril. & horas). La IGIV es & administr & Asp \\
\hline Seguidan & en los 10 & isolona a & dipiridamol \\
\hline pasa a ac & prin & altas dosis 3 & administrarán \\
\hline antitromk & enfermedad (5to y 7 to & vía i.v., durante 2-3 & durante 1 año 0 \\
\hline $5 \mathrm{mg} / \mathrm{kg} / \mathrm{dí} a$ & pacientes que & asociados & la resolución \\
\hline durante & no mejoran o que & ciclosporir & \\
\hline
\end{tabular}




\begin{tabular}{|c|c|c|c|}
\hline $\begin{array}{l}\text { semanas más o } \\
\text { hasta que la cifra } \\
\text { de plaquetas se } \\
\text { normalice }\end{array}$ & $\begin{array}{l}\text { tengan una recaída } \\
\text { recibirán otra dosis de } \\
\text { IGIV.Una segunda } \\
\text { dosis de IVIG debe ser } \\
\text { administrada a las 36- } \\
48 \text { horas en pacientes } \\
\text { que no muestran una } \\
\text { mejoría clínica }\end{array}$ & & $\begin{array}{l}\text { del aneurisma } \\
\text { coronario }\end{array}$ \\
\hline $\begin{array}{l}\text { EL AAS a bajas } \\
\text { dosis es la terapia } \\
\text { ideal para los } \\
\text { aneurismas en los } \\
\text { vasos de pequeño } \\
\text { y medio calibre, } \\
\text { junto ron } \\
\text { clopidogrel o } \\
\text { ticlopidina. }\end{array}$ & $\begin{array}{l}\text { A veces la IGIV puede } \\
\text { dar problemas de } \\
\text { cefalea, meningitis } \\
\text { aséptica }\end{array}$ & $\begin{array}{l}\text { No obstante, esta } \\
\text { alternativa no debe } \\
\text { considerarse definitiva } \\
\text { y serán necesarios } \\
\text { ulteriores estudios para } \\
\text { confirmar la indicación } \\
\text { de los esteroides en los } \\
\text { casos de EK resistentes } \\
\text { a la } \\
\text { IGIV }\end{array}$ & $\begin{array}{l}\text { reposo en cama } \\
\text { durante las 2-3 } \\
\text { primeras semanas de } \\
\text { enfermedad ó } 72 \\
\text { horas después de } \\
\text { ceder la fiebre por el } \\
\text { riesgo de infarto de } \\
\text { miocardio en la fase } \\
\text { aguda. }\end{array}$ \\
\hline $\begin{array}{l}\text { Cuando los } \\
\text { aneurismas son } \\
\text { grandes y de } \\
\text { expansión rápida } \\
\text { puede haber un } \\
\text { alto riesgo de } \\
\text { trombosis, por lo } \\
\text { que deben ser } \\
\text { manejados con } \\
\text { heparina } \\
\text { intravenosa } \\
\text { para } \\
\text { tratamiento a } \\
\text { largo plazo deben } \\
\text { ser manejados } \\
\text { con Warfarina }\end{array}$ & $\begin{array}{l}\text { reduce el estrés } \\
\text { oxidativo que se } \\
\text { produce en los vasos } \\
\text { sanguíneos en la fase } \\
\text { aguda de la EK }\end{array}$ & $\begin{array}{l}\text { metilprednisolona: } 30 \\
\mathrm{mg} / \mathrm{kg} \text { una vez al día por } \\
1-3 \text { días }\end{array}$ & \\
\hline
\end{tabular}

Fuente: Protocolos diagnósticos y terapéuticos: Alfonso Delgado Rubio y Enfermedad de Kawasaki: diagnóstico y tratamiento: Margarita Cardozo López et al. 


\section{DISCUSIÓN}

Patogénicamente se asocia a un riesgo significativo de desarrollo de anomalías de las arterias coronarias si no se trata. (17). Estudios recientes sobre KD revelaron daño endotelial y la generación de trombina resultante, así como la activación de las células $B$ durante la fase aguda (18). La causa es desconocida, pero, se sospecha que es provocada por un patógeno infeccioso no identificado en niños genéticamente predispuestos; modelos experimentales en animales han demostrado el papel fundamental de la IL-1 $\beta$ en la patogénesis de esta enfermedad y el potencial terapéutico de dirigirse a esta vía (19).

Así también otros autores sugieren interacción de factores de susceptibilidad genética (que incluyen: riesgo de concordancia en gemelos idénticos en $13 \%$, mayor incidencia de EK en niños cuyos padres tienen antecedentes de EK y mayor incidencia de EK en hermanos de pacientes afectados) (20) y ambientales con desencadenantes infecciosos, seguida de una respuesta inmune anormal posterior caracterizada por niveles elevados de citocinas y quimiocinas inflamatorias durante la fase aguda. Se ha acumulado evidencia que sugiere que un desequilibrio entre las células T auxiliares 17 (Th17s) y las células T reguladoras (Tregs) está asociado con respuestas inmunes aberrantes en la EK (21).

El tratamiento de la enfermedad aguda es la inmunoglobulina intravenosa y aspirina, especialmente dentro de los 10 días posteriores al inicio de la enfermedad lo cual ha demostrado disminuir la incidencia de aneurismas coronarios a menos de 5\%. (22), (23), sobre todo en fase subaguda, donde provocan mayores complicaciones principalmente cardiovasculares como los aneurismas (24); la eficacia de este tratamiento en reducir los síntomas de inflamación y en prevenir el desarrollo de alteraciones coronarias. (25)

Si no hay respuesta al tratamiento, los pacientes reciben una segunda dosis de inmunoglobulina intravenosa con $o$ sin corticosteroides $u$ otros tratamientos complementarios (26). Las terapias con corticosteroides y otros fármacos inmunosupresores son alternativas utilizadas en el tratamiento de la enfermedad de Kawasaki resistente a inmunoglobulina (27).

Como se dijo una vez demostrada la resistencia a inmunoglobulinas intravenosas, se han propuesto tratamientos alternativos ya mencionados con uso tradicional de corticoides, como los inhibidores del TNF-alfa, y todos los pacientes con aneurismas coronarios, deben realizar la evaluación periódica de la perfusión miocárdica (28).

También se ha demostrado que el uso de glucocorticoides se considera como terapia preventiva para niños con EK grave y como terapia de rescate para quienes no responden inicialmente. Sin embargo, el uso rutinario de glucocorticoides en la EK sigue siendo un tema polémico y necesitaría más estudios (29), (30).

Respecto a las dosis del tratamiento con metilprednisolona $30 \mathrm{mg} / \mathrm{kg}$ una vez al día por 1 - 3 días, ácido acetilsalicílico $100 \mathrm{mg} /$ día, una dosis única de inmunoglobulina IgG intravenosa 
$2 \mathrm{~g} / \mathrm{kg}$ y terapia dialítica interdiaria, logrando una recuperación completa (31). Respecto a la terapia biológica, especialmente con rituximab, es una alternativa terapéutica eficaz (32).

\section{CONCLUSIONES}

La enfermedad de Kawasaki comprende complicaciones que acarrean aneurismas y que en algunos niños que no reciben tratamiento provocan cardiopatía isquémica e infarto de miocardio. Los factores genéticos y ambientales se han relacionado con la patogenia, sin embargo, no está clara su etiología, existe una respuesta inmune anormal caracterizada por niveles elevados de citocinas y quimiocinas inflamatorias durante la fase aguda. Por esto un pilar fundamental es realizar un diagnóstico oportuno y tratamiento que incluye la terapia combinada de ácido acetilsalicílico más inmunoglobulina $G$, la cual reduce la incidencia de aneurismas coronarios, sin embargo, los corticosteroides también han demostrado su eficacia especialmente cuando la terapia anterior es resistente.

Posteriormente al tratamiento agudo o subagudo se debe realizar seguimiento para controlar su salud cardíaca regularmente, repetir ecocardiograma mensualmente durante 6 meses hasta la resolución. El tratamiento de las complicaciones cardíacas depende del tipo de afección cardíaca presente. Si un aneurisma de la arteria coronaria se rompe, el tratamiento puede incluir fármacos anticoagulantes, colocación de stent o cirugía de derivación.

\section{Referencias}

1. Ana Barios Tascón FCMHRSAFCJSMJPLP. Consenso nacional sobre diagnóstico, tratamiento y seguimiento cardiológico de la enfermedad de Kawasaki. Asociación Española de Pediatría. 2018; 89(3): p. 188.e1-188e22.

2. Sánchez-Manubens J. Enfermedad de Kawasaki. Sociedad Española De Reumatología Pediátrica. 2020;: p. 213-224.

3. Carolina Mejía Arens NSONSC. Actualización en enfermedad Kawasaki en población pediátrica. Rev Med Sinergia. 2020 Junio;: p. 5.

4. Luis Martín Garrido-García SGAPCFGRUGMAYN. Enfermedad de Kawasaki en lactantes bajo un año de edad. Un reto para el diagnóstico y tratamiento de los pacientes. Experiencia en un centro hospitalario en México. Revista chilena de infectología. 2020; 37(5).

5. Gómez LML. Enfermedad de Kawasaki. Pediatría U. De Antioquia. 2016.

6. Eduardo Marín-Hernández AAVFMSAYLDS. Enfermedad de Kawasaki. Comunicación de dos casos. Rev Cent Dermatol Pascua. 2018 Enero-Marzo; 27(1). 
7. Elisa Fernández-Cooke ABTJALCDGLJSMCC. Infecciones previas o coincidentes con la sospecha de enfermedad de Kawasaki ¿debemos cambiar nuestra actitud? Anales de Pediatría. 2019; 90(4): p. 213-218.

8. Santos-Calderón LA RHKRGUGBMLCGQHALDVGSESNJLHJ. Enfermedad de Kawasaki: Conceptos actuales. Avan C Salud Med. 2018;: p. 17-26.

9. Shreya Agarwal DKA. Enfermedad de Kawasaki: etiopatogenia y nuevas estrategias de tratamiento. Experto Rev Clin Immunol. 2017 Marzo; 3(13): p. 247-258.

10. Magali Noval Rivas MA. Enfermedad de Kawasaki: fisiopatología y conocimientos de modelos de ratón. Nat Rev Rheumatol. 2020 Julio; 7(16): p. 391-405.

11. Paul Brogan JCBJCVDDEJBGHHGTWJMLIMPMRMOMRMT. Manejo cardiovascular de por vida de pacientes con enfermedad de Kawasaki previa. Heart. 2020;: p. 411-420.

12. Kara J Denby DECLWM. Manejo de la enfermedad de Kawasaki en adultos. Heart. 2017 Julio 27; 103(22): p. 1760-1769.

13. Coria LJ RBSCDLECUGRRMMDTJJRLEPGC. Complicaciones cardíacas en niños mexicanos con enfermedad de Kawasaki. Estudio multicéntrico de la red Kawasaki de México. Rev Latin Infect Pediatr. 2018; 3(31): p. 110-117.

14. Garrido-García LM SBJERF. Kawasaki disease: clinical features, laboratory and coronary artery lesions. Acta Pediatr Mex. 2015; 4(36): p. 314-321.

15. Morales-Quispe JA EZNCCRGLJRQJBRE. Evolución clínica y complicaciones cardiovasculares en niños con enfermedad de Kawasaki. Rev Med Inst Mex Seguro Soc. 2011; 3(49): p. 295-300.

16. Brian W. McCrindle BC. El papel de la ecocardiografía en la enfermedad de Kawasaki. Int J Rheum Dis. 2018;: p. 50-55.

17. Ankur Kumar Jindal RKPAPSGSS. Enfermedad de Kawasaki: características, diagnóstico y presentaciones inusuales. Expert Rev Clin Immunol. 2019 Octubre; 10(15): p. 1089-1104.

18. Sakurai Y. Aspectos autoinmunes de la enfermedad de Kawasaki. J Investig Allergol Clin Immunol. 2019; 4(29): p. 251-261.

19. Noval Rivas MyMA. Enfermedad de Kawasaki: fisiopatología y conocimientos de modelos de ratón. 2020 mayo; Nature Reviews Reumatología volumen 16 (16).

20. Eiliin Rife ea. Enfermedad de Kawasaki: una actualización. Informes actuales de reumatologia. 2020 Septiembre; 22(75). 
21. Kazunari Kaneko SA,YA,TKyST. Nuestra comprensión evolutiva de la patogenia de la enfermedad de Kawasaki: papel de la microbiota intestinal. Frontiers inmunology. 2020 julio 24; 16(1616).

22. Viviana Molina Alpízar BUA. Enfermedad de Kawasaki. Medicina Legal de Costa Rica. 2015; 32.

23. Olano-Ochoa C. ENFERMEDAD DE KAWASAKI CON AFECTACIÓN DE ARTERIAS CORONARIAS. ReV Experiencia en Medicina Del Hospital Regional Lambayeque. 2017; 3(3).

24. Herrera Quinde I. Tratamiento y complicaciones de la enfermedad de kawasaki en pacientes pediátricos. Repositorio Universidad de Guayaquil. 2016.

25. Luisa Berenise Gámez-Gonzáles MAYN. Enfermedad de Kawasaki: un vistazo al cincuento. Rev Invest Med Sur Mex. 2015; 4(22): p. 169-181.

26. Aaron Saguil MFSG. Diagnóstico y tratamiento de la enfermedad de Kawasaki. Am Fam Physician. 2015 Marzo 15; 6(91): p. 365-71.

27. Hellmann Adrián Escobar GMGJEIPHMTMJAMBJFVMCFCGAACB. Tratamiento farmacológico de la enfermedad de Kawasaki. Universidad Nacional de Colombia. 2019 Enero 1; 67(1).

28. Sabrina Bressieux-Degueldre DSMHNSFV. Enfermedad de Kawasaki: una actualización. Rev Med Suisse. 2018 Febrero 14;: p. 384-389.

29. Miura M. Papel de los glucocorticoides en la enfermedad de Kawasaki. Int J Rheum Dis. 2018 Enero; 1(21): p. 70-75.

30. Escobar HA,MGG,IPJE,TMHM,MBJA,VMJF,CGCF,\&CB. Tratamiento farmacológico de la enfermedad de Kawasaki. Revista de la Facultad de Medicina. 2019; 1(67): p. 103-108.

31. Jacklyn Cristina Guzmán Montealegrea LMSRACRCMHV. Enfermedad de Kawasaki. Reporte de un caso infrecuente en el adulto. Revista Colombiana de Reumatología. 2018; 26(2): p. 132-136.

32. J. Mestre FMVRS. Vasculitis de mediano vaso. Vasculitis necrotizantes: panarteritis nodosa y vasculitis ANCA asociadas. Enfermedad de Kawasaki. Medicine - Programa de Formación Médica Continuada Acreditado. 2017;: p. 1704-1715. 